\title{
Indexing Intelligence using Benchmark Classifier
}

\author{
Mouneshachari ${ }^{1}$, Sanjay Pande $\mathbf{M}^{2}$ \\ ${ }^{1}$ Department of Computer Science \& Engineering, Jain University, India \\ ${ }^{2}$ Department of Computer Science \& Engineering, GMIT, India
}

\begin{tabular}{l}
\hline \hline Article Info \\
\hline Article history: \\
Received Jun 11, 2019 \\
Revised Sep 12, 2019 \\
Accepted Oct 3, 2019 \\
\hline
\end{tabular}

Keywords:

Classsification

EEG analysis

Human intelligence index

$\mathrm{K}$ nearest neighbour

Machine learning

\begin{abstract}
Human being as a parameter for assessment is a complex component for any researcher, since the field of medical sciences opens up too many unsolved queries. In this context if emotions are to be quantified it involves both scientific and certain Non-scientific issues. In terms of medical concept Electroencephalogram (EEG) helps in understanding specific regions of the brain. Since functional capabilities of regions of the brain can be understood by the probes attached to that particular region which intern provides the electrical responses. In the present work, It has been tried to encapsulate the signals in EEG to create an Index of quantification to understand the basic feelings of emotion of an Individual. As a researcher perspective to deduce any mathematical equation, a benchmark data is a major requirement. Hence to enumerate the algorithm, a specific classification model using k-NN has been taken up which enables to understand the similarity and dissimilarity factor of the recorded signals of an individual with the benchmark data.
\end{abstract}

Copyright $@ 2020$ Institute of Advanced Engineering and Science. All rights reserved.

\section{Corresponding Author:}

Mouneshachari S,

Departmet of Computer Science \& Engineering,

GM Institute of Technology,

PB No. 4, P B Road, Davangere, Karnataka, India.

Email: mounesh_s@yahoo.co.in

\section{INTRODUCTION}

The most unpredictable organ in human body is the brain and its functions. One of the approach for analysis of these functions is EEG interpretation. EEG analysis has become one of the trending topic in the area of brain functional analysis through computing techniques. EEG provides non-invasive method of capturing the data or information of the brain functions. Psycological perspectives of human behavior analysis has produced ocean of conclusions corresponding to brain functions. The accuracy and efficiency of these conclusions is still left open for the researchers. Intelligence Quotient (IQ) is one of the readily available psychological measure to quantify Human intelligence. It has number of revisions in its mathematical model since 1910, but concluded during the year 1964. Intelligence Quotient (IQ) is a measure of intelligence of an individual as a ratio of mental age [1] with the chronological age. Several researchers proposes another measure during the 1980s called as Emotion Quotient (EQ). They say that IQ alone cannot quantify human intelligence, there can be another measure for social behavior which may contribute for human intelligence. Emotion Quotient is a newly invented measure for the degree of success of an individual. In this concern, several models [2-6] are available for both IQ and EQ estimations, but they may be inefficient in providing the trueness of properties that is, the subject may be attempted to answer objective type questions without any analysis or thinking.

The measures used to quantify human intelligence are psychological models, these may or may not provide efficient quantification. Efficient quantification of IQ and EQ of any subject deals with the trueness of the assessment of the subject while answering each question or item from the given scale. Here IQ and EQ are the psychological models and these are assessed based on the questionnaire (through the corresponding scales). Psychological models may fail to ensure the efficiency of the assessment and hence may lead to 
improper analysis of subject intelligence. Also there is a need of mathematical or any computing model to combine these IQ and EQ parameters to derive Human Intelligence.

In order to improve the efficiency of these IQ and EQ parameters, this paper is trying to include the brain functionalities during the assessment of both IQ and EQ and then trying to combine using classification techniques to derive a new and revolutionary index called as Human Intelligence Index (HII). In this paper, own EQ model is used to verify the functionalities of the proposed model to quantify HII. This paper mainly deals with the feature extraction through the efficient estimation of IQ and EQ, then the construction of knowledge base through K-means clustering and utilizing the same for classification using k-NN classifier to quantify Human Intelligence Index.

There are several classification techniques available in the literature, namely Support Vector Machine (SVM), Artificial Neural Network (ANN), Probabilistic Neural Network (PNN), k-Nearest Neighbor (k-NN), Hidden Markov Model (HMM) and so on. The above mentioned classification techniques will have their own strengths and weaknesses. One of the simplest, efficient and supervised machine learning classification technique, when the number of clusters are known is k-NN (k Nearest Neighbor) [7]. EEG signal is captured using NeuroSky [8] device through a open source software OpenVibe [9], the captured signals are processed for their Sphericity (similarity), IQ and EQ estimations are conducted, Knowledge Base construction using kmeans and classification of the test sample using k-NN are implemented in MATLAB R2013a Student Version [10-11].

HII is a classified combination of IQ (Intelligence Quotient) and EQ (Emotion Quotient) and may be defined as one of the four different forms that is Survive, Success, Satisfied and Supreme along with quantified entity [12]. This may declare the type of the test sample and its rating within the class. Obviously this parameter should be seriously considered to differentiate the sample which has made a right attempt during the assessment.

\section{RELATED WORK}

\subsection{Intelligence Quotient (IQ) \& Emotion Quotient (EQ)}

A psychological approach for the assessment of cognitive intelligence since many centuries is IQ model. Later with the number of revisions and new approaches, this model took number of shapes and finally landed to a mathematical model that is the ratio of mental age and chronological age as shown in (1).

$$
\mathrm{IQ}=(\text { Mental Age }(\mathrm{MA}) / \text { Chronological Age }(\mathrm{CA}))^{*} 100
$$

Since 1970s experts working on other parameters which may be contributing or affecting measure of intelligence. Some of the studies [13] have shown that each side (left and right) of the brain processes the information differently and produces distinct characteristics that is, left side of the brain performs the tasks related to logic, analytical thought, Science \& Mathematics and language, where as right-side processes holistic thought, creativity, intuition, emotion and so on. In this regard several psychological models are available to quantify both IQ and EQ in the literature. Certain studies have shown that IQ is not only the parameter for overall growth of any individual in the environment, also there may be number of other parameters involved related to social and ethical concerns [14].

\subsection{EEG Analysis}

One of the easiest resource for brain function analysis is Electroencephalogram (EEG). These days EEG has a wide variety of applications and research openings in all areas [15]. One of the study [16] lists several versions of wearable EEG capturing equipments ranging from 28 to 256 channels along with output signal efficiency and characteristics. Indeed, as the technology grows, the efficiency, characteristics and the cost of the equipments should be improved. Hence the device NeroSky provides a technical resources in the form of single channel, filter and digitally recordable EEG signal during capture. The study have shown that the detection through recognition of pattern from the EEG signal when a particular item of a scale is provided for response collection from more than one subject [17]. The paper [18] has tried to design neuroprosthetics limb by adopting the analysis of EEG signals. Here author analyses the EEG signals to study the percentage of Meditated and Attention mind. This percentage has been used as a input to provide a movement to artificial limb.

\section{3. k-NN Classifier}

Utilizing collected data through processing, analysis for results and drawing proper inferences are the major roles of Computer Science and Engineering. In this concern, a suitable classifier is required, k-NN is a simple classifier, suitable when the number of classes are known, efficient when suitable distance finding 
algorithm is used and can be combined [19-23] with other classifiers to define hybrid classifiers for the effective computations. Since k-NN is one of the supervised Machine Learning Technique, it is basically used for labeling the test sample with reference to knowledge base [24]. The paper [25] declares the performance of $\mathrm{k}-\mathrm{NN}$ classifier is upto $99.9554 \%$ for Kelberg dataset when compared to other classifiers.

\section{RESEARCH METHOD}

Figure 1 shows the proposed methodology of the paper. Here the proposed system functions in two different phases, learning (Training) and quantification (Testing) phases. Knowledge Base construction is the major objective of learning phase, the next phase performs classification and quantification of HII of test sample by utilizing knowledge base.

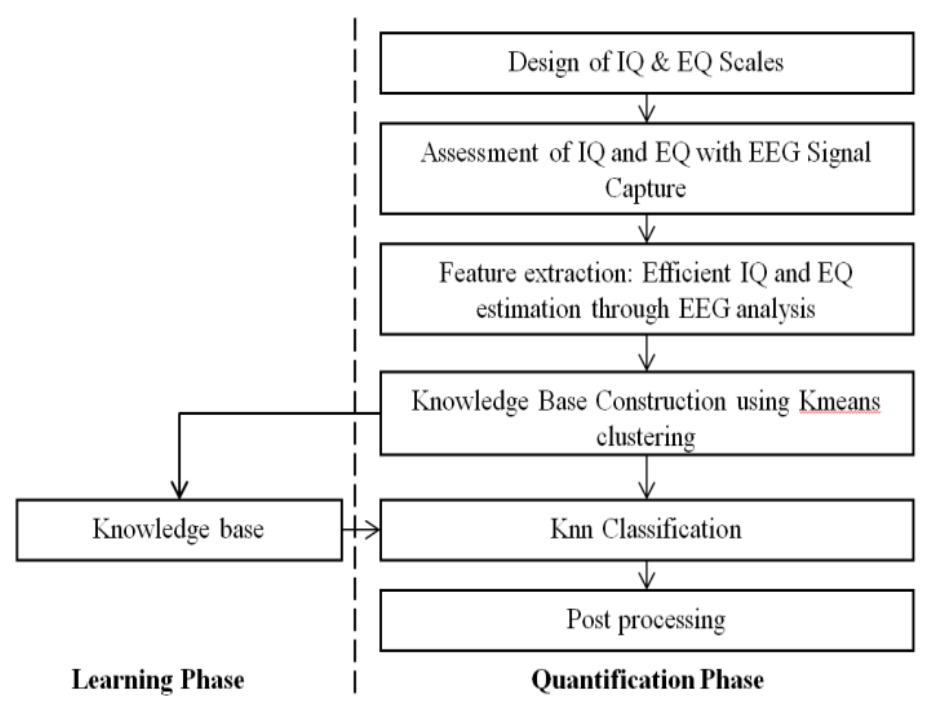

Figure 1. Methodology for an efficient estimation of Human Intelligence Index

\subsection{Learning Phase}

\subsubsection{Design of IQ and EQ Scales}

The research work has considered Intelligence Quotient and Emotion Quotient as features / parameters for the HII classification. For the assessment of IQ and EQ from the subjects, a well defined and designed IQ and EQ scales are essential and they are prepared based on the functionalities of the brain. These scales are verified for validity and reliability by using Cronbach's alpha test model [26].

\subsubsection{Assessment of IQ and EQ with EEG Signal Capture}

Around fifty subjects concerned to engineering domain with same environment are considered and trained as per the requirement under the expert supervision. Figure 2 shows the experiment conducted for the collection of EEG capture during the assessment. Neurosky, a single channel EEG capture device is employed to capture the EEG. The captured EEG signal for each item of a scale is stored in the form of file. This process is continued for all the items of the IQ and EQ scales corresponding to one particular subject. The captured EEG signal is used to verify the trueness of assessment of each item which is called as validationQuotient of that particular item assessment. The computation of validationQuotient is described in paper [17]. The above steps are followed for all other items of IQ and EQ scales corresponding to a particular subject.

The final assessment of the item 1 is estimated using the (2).

FinalIQItem1 = AnsIQItem1 * validationQuotient 


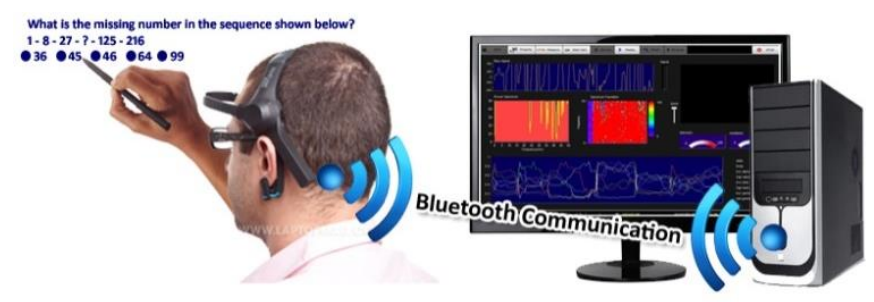

Figure 2. Brain signal capture during IQ or EQ assessment of subject

\subsubsection{Feature extraction}

The designed IQ and EQ scales consisting of ten items. Estimation of HII mainly involves the classification by considering IQ and EQ as factors or features. IQ is estimated using the (3).

$$
\mathrm{IQ}=\mathrm{MA} / \mathrm{CA} * 100
$$

Where MA is mental age and CA is chronological age, MA is given by the (4).

$$
\text { MA = finalIQitem1+finalIQitem2+........finalIQitem10 }
$$

Here finalIQitem1, finalIQitem2 upto finalIQitem10 are the true assessments estimation using the (2).

CA is a chronological age and it is given by the (5).

$$
\mathrm{CA}=(\mathrm{MA} 1+\mathrm{MA} 2+\ldots \ldots . .+\mathrm{MAn}) / \mathrm{n} ;
$$

Where MA1, MA2.MAn are the mental ages of $\mathrm{n}$ subjects involved in the process.

The proposed work uses the own designed EQ model for the estimation of Emotion Quotient (EQ). The EQ of any subject is given by the (6).

$$
\mathrm{EQ}=(\text { finalEQitem } 1+\text { finalEQitem } 2+\ldots .+ \text { finalEQitem } 10) / \operatorname{maxEQ}
$$

Where, maxEQ is the maximum score of assessment. This research work has considered around 50 subjects for the knowledge Base construction. Hence the above IQ and EQ estimation steps are followed for all the subjects. Table 3 shows the normalized IQ and EQ parameters of 50 samples. Survive (C1), Success(C2), Satisfied(C3) and Supreme(C4) clusters as shown in Figure 3.

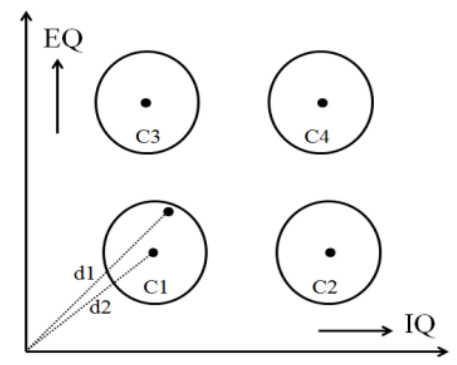

Figure 3. Survive (C1), Success(C2), Satisfied(C3) and Supreme(C4) clusters

\subsubsection{Learning Phase (Knowledge Base (S-Quotient Base) Construction):}

Since, no standard dataset is available for the classification of HII, k-means an unsupervised technique is employed for the clustering of samples called Knowledge Base. Based on the Romain Rolland [27] approach, HII is identified in four different indices namely Survive, Success, Satisfied and Supreme. Table 3 Shows the Knowledge Base for the HII classification \& Figure 3 shows the four clusters when mapped with respect to IQ and EQ. 


\subsubsection{Quantification/Testing phase}

A subject (test sample), whose HII has to be estimated will be made to take IQ and EQ assessments and estimations. k-NN, a supervised classifier is applied by involving the constructed Knowledge Base and the test sample's IQ and EQ parameters. k-NN classifier labels the test sample.

\subsubsection{Post processing}

For better classification of the test sample, a second level analysis has been imparted in this research work by including a concept called Cluster Quotient (CQ). Here CQ states the status of the test sample within the cluster. Through this factor one can easily find out the development or improvement of the test sample across the age. CQ is given by the (7).

$$
\mathrm{CQ}=(\mathrm{d} 1-\mathrm{d} 2) * 100
$$

Where $\mathrm{d} 1$ is the distance from origin to sample and $\mathrm{d} 2$ is the distance from origin to centroid as shown in Figure 3. The formulation of HII is done by using the label of the test sample followed by its corresponding cluster quotient as shown in (8).

$$
\mathrm{HII}=\text { Class_label CQ }
$$

\section{RESULTS}

Table 1 shows estimated IQ's of five subjects (for demonstration purpose in the paper) in both traditionally available method and the proposed efficient method using EEG analysis.

\begin{tabular}{|c|c|c|c|c|c|}
\hline Subject & $\begin{array}{c}\text { Traditional } \\
\text { Method }\end{array}$ & IQ & $\begin{array}{c}\text { Proposed } \\
\text { Method }\end{array}$ & Efficient IQ & Difference \\
\hline 1 & $7 *$ & $7 / 6.6=106$ & $4.56^{* * *}$ & $4.56 / 3.798=120$ & 14 \\
\hline 2 & 5 & $5 / 6.6=75$ & 4.69 & $4.69 / 3.798=123$ & 48 \\
\hline 3 & 8 & $8 / 6.6=122$ & 2.22 & $2.22 / 3.798=58$ & 64 \\
\hline \multirow[t]{2}{*}{4} & 4 & $4 / 6.6=61$ & 3.32 & $3.32 / 3.798=87$ & 26 \\
\hline & 9 & $9 / 6.6=137$ & 4.2 & $4.2 / 3.798=110$ & 27 \\
\hline
\end{tabular}

Table 1. Comparison of IQ Estimation of Both Traditional and Proposed Methods on any Five Samples

*7 is the Mental Age of subject 1 and 6.6 is the Chronological Age that is the average score of all the subjects in the assessment process. Difference between these IQ's are the actual efficient evaluator to verify the trueness of the individual scores during the assessment. These estimations are shown below.

* In traditional method, total IQ quantity is estimated by summing the correct answers in the IQ scale. Example is

$1+1+0+0+1+1+1+1+1+1$, here 0 and 1 indicates wrong and correct answers respectively.

** In the proposed method there are two types of mental age (MA) estimations

a) Method 1: Considering wrong answers (without multiply by evaluated answer) This method considers the person who might have analyzed the question while marking the wrong answers.

$$
0.2811 .5376+0.2332+0.6512+0.9153+0.5654+0.8176+0.6381+0.3324+0.1457=5.45
$$

b) Method 2: Without considering wrong answers (Apply multiplication with evaluated answer that is 0 and 1 for wrong and right answers respectively). This method do not considers the person who might have analyzed the question while marking wrong answer.

$$
1 \times 0.2811+1 \times 0.5376+0 \times 0.2332+0 \times 0.6512+1 \times 0.9153+1 \times 0.5654+1 \times 0.8176+1 \times 0.6381+
$$
$1 \times 0.3324+1 \times 0.1457=4.56$

The Chronological Age (CA) can be estimated as

Traditional method $\mathrm{CA}=(7+5+8+4+9) / 5=6.6$

Proposed method CA $=(4.56+4.69+2.22+3.32+4.2) / 5=3.798$

Table 2 shows the EQ estimation in both traditional and proposed method. Since EQ scale is designed on five point likert scale, the scores of each item can be summed to find the total score of an 
individual in the assessment. 36 is the total score of subject 1 in traditional method where as 25.1385 is the total score in the proposed method when EEG analysis is conducted on each item assessment. There are totally ten items in the considered EQ scale and each item maximum score is five hence total score of the scale will be 50 . Hence the EQ is estimated as the individual score divided by the total score of the scale. Similarly ther difference shows the trueness of the individual assessment. Table 3 shows the normalized EQ and IQ values of fifty subjects.

Table 2. Comparison of EQ Estimation of Both Traditional and Proposed Methods on any Five Samples

\begin{tabular}{cccccc}
\hline Subject & $\begin{array}{c}\text { Traditional } \\
\text { Method }\end{array}$ & EQ & $\begin{array}{c}\text { Proposed } \\
\text { Method }\end{array}$ & Efficient EQ & Difference \\
\hline 1 & $36^{*}$ & $36 / 50=0.72$ & $25.14^{* *}$ & $25.14 / 50=0.50$ & 0.28 \\
2 & 24 & $24 / 50=0.48$ & 46.26 & $46.26 / 50=0.93$ & 0.46 \\
3 & 49 & $49 / 50=0.98$ & 12.98 & $12.98 / 50=0.26$ & 0.72 \\
4 & 33 & $33 / 50=0.66$ & 5.28 & $5.28 / 50=0.11$ & 0.55 \\
5 & 47 & $47 / 50=0.94$ & 48.25 & $48.25 / 50=0.97$ & 0.03 \\
\hline
\end{tabular}

*In traditional method, summing up of all the scores of the items from the EQ scale.

Example: $3+5+4+3+4+4+3+5+2+3=36$

**In proposed method, each score of the item is multiplied with maximum sphericity of the EEG signal of that particular item.

Example: $3 \times 0.6512+5 \times 0.9342+4 \times 0.5589+3 \times 0.1982+4 \times 0.8978+4 \times 0.8734+3 \times 0.5392+$ $5 \times 0.9127+2 \times 0.3278+3 \times 0.5874=25.1385$

Table 3. Estimated Efficient IQ and EQ Based on the Responses from the Subject

\begin{tabular}{cccccccccccc}
\hline Sample & IQ & EQ & Label & Sample & IQ & EQ & Label & Sample & IQ & EQ \\
\hline S1 & 0.91 & 0.34 & Success & S18 & 0.87 & 0.9 & Supreme & S35 & 0.33 & 0.21 & Survive \\
S2 & 0.78 & 0.77 & Supreme & S19 & 0.66 & 0.95 & Supreme & S36 & 0.66 & 0.16 & Survive \\
S3 & 0.88 & 0.21 & Success & S20 & 0.11 & 0.16 & Supreme & S37 & 0.92 & 0.9 & Success \\
S4 & 0.76 & 0.89 & Supreme & S21 & 0.17 & 0.13 & Survive & S38 & 0.43 & 0.98 & Supreme \\
S5 & 0.85 & 0.13 & Success & S22 & 0.87 & 0.11 & Survive & S39 & 0.54 & 0.24 & Satisfied \\
S6 & 0.79 & 0.22 & Success & S23 & 0.32 & 0.33 & Success & S40 & 0.56 & 0.13 & Survive \\
S7 & 0.35 & 0.87 & Satisfied & S24 & 0.43 & 0.43 & Survive & S41 & 0.99 & 0.29 & Survive \\
S8 & 0.32 & 0.67 & Satisfied & S25 & 0.1 & 0.19 & Survive & S42 & 0.18 & 0.23 & Success \\
S9 & 0.95 & 0.2 & Success & S26 & 0.12 & 0.89 & Survive & S43 & 0.15 & 0.24 & Survive \\
S10 & 0.99 & 0.67 & Supreme & S27 & 0.24 & 0.94 & Satisfied & S44 & 0.88 & 0.88 & Survive \\
S11 & 0.93 & 0.15 & Success & S28 & 0.32 & 0.85 & Satisfied & S45 & 0.41 & 0.25 & Supreme \\
S12 & 0.78 & 0.14 & Success & S29 & 0.9 & 0.8 & Satisfied & S46 & 0.93 & 0.98 & Survive \\
S13 & 0.76 & 0.87 & Supreme & S30 & 0.2 & 0.22 & Supreme & S47 & 0.88 & 0.29 & Supreme \\
S14 & 0.85 & 0.24 & Success & S31 & 0.94 & 0.87 & Survive & S48 & 0.91 & 0.76 & Success \\
S15 & 0.2 & 0.99 & Satisfied & S32 & 0.67 & 0.98 & Supreme & S49 & 0.87 & 0.27 & Supreme \\
S16 & 0.34 & 0.3 & Survive & S33 & 0.93 & 0.9 & Supreme & S50 & 0.32 & 0.24 & Success \\
S17 & 0.87 & 0.78 & Success & S34 & 0.24 & 0.19 & Supreme & & \\
\hline
\end{tabular}

Table 4 shows the four different clusters/classes and their centroids. These clusters acts as a knowledge base for further test sample classification. Figure 4 shows the centroids of clusters with the labeling as Survive, Success, Satisfied and Supreme. These classes are defined as per Romain Rolland Commitment \& Competency based classification.

Figure 5(a) depicts the classification of a test sample1 whose $\mathrm{IQ}=0.24$ and $\mathrm{EQ}=0.15, \mathrm{k}-\mathrm{NN}$ is applied on the Table 3 including the test sample 1, it labels the test sample as class 1 that is Survive, this is because the efficient EQ and IQ's are limited (less than the average).

$$
\mathrm{CQ}=(0.2830 / 0.3755) * 100=75
$$

Table 4. Centroids of Clusters (Knowledge Base)

\begin{tabular}{ccc}
\hline Cluster & IQ & EQ \\
\hline Centroid 1 & 0.52 & 0.31 \\
Centroid 2 & 0.87 & 0.36 \\
Centroid 3 & 0.39 & 0.86 \\
Centroid 4 & 0.81 & 0.78 \\
\hline
\end{tabular}




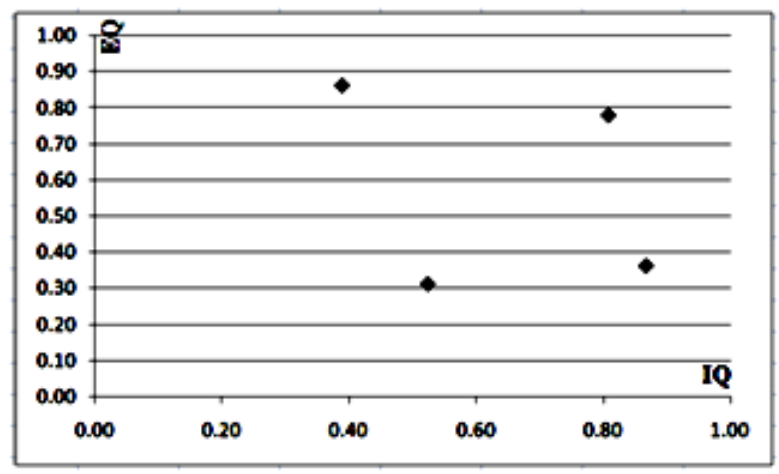

Figure 4. Survive, success, satisfied and supreme classes

This indicates that test sample 1 belongs to survive class and the 75 is the Cluster Quotient of the test sample within the cluster. The overall Human Intelligence Index is given by Survive 75 for test sample 1. Similarly Figure 5(b) depicts the classification of test sample 2 with $\mathrm{IQ}=0.85$ and $\mathrm{EQ}=0.30 \mathrm{k}-\mathrm{NN}$ labels the test sample 2 to class 4 that is, Supreme class and CQ $=102$. Test sample 2 is classified as Supreme class and 102 is the Cluster Quotient. These can be put together to define HII as Supreme 102.

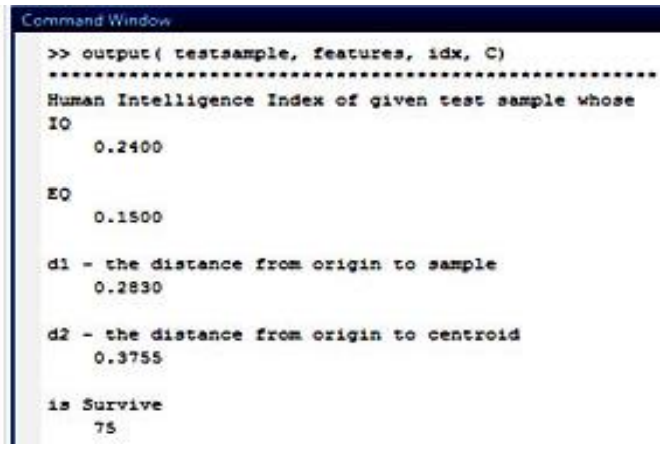

(a)

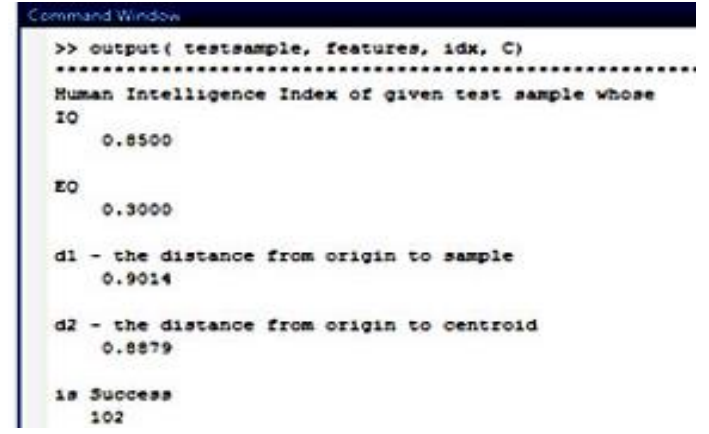

(b)

Figure 5. (a) MATLAB output for the Survive case study, (b) Success case study

\section{c) Confusion matrix}

Table 5 depicts the confusion matrix for the proposed classifier by involving 80 samples in the process. Here around 22 samples actually belongs to survive class, around 19 samples predicted as Survive. Similarly 19, 18 and 21 were actual samples corresponding to classes, but 19,19 and 23 are predicted as Success, Satisfied and Supreme respectively. Hence the accuracy of the classifier is $87.5 \%$.

Table 5. Confusion Matrix for the Proposed Classifier

\begin{tabular}{|c|c|c|c|c|c|}
\hline & Survive & Success & Satisfied & Supreme & Actual \\
\hline Survive & 18 & 2 & 0 & 2 & 22 \\
\hline Success & 0 & 17 & 2 & 0 & 19 \\
\hline Satisfied & 0 & 0 & 16 & 2 & 18 \\
\hline Supreme & 1 & 0 & 1 & 19 & 21 \\
\hline Predicted & 19 & 19 & 19 & 23 & 80 \\
\hline
\end{tabular}

\section{CONCLUSION}

Any distribution will have a mixture of different parameters presenting its validity for the presence in a dataset. In the present work a highly contradicting organ of the body called brain and its functionalities for the behavior of an individual is presented to corroborate the working model with any data a specific class of data has been used which enables to illustrate the various classifying factors that has been considered and four terminologies namely Survive, Success, Satisfied and Supreme respectively are presented as the four 
regions and factors for quantification of Human Intelligence. The process of Indexing has enabled to restrict the overlapping factors in different regions. The main focus of this paper is to design and verify the computing model, hence the knowledge base used for classification is designed by considering certain simulated data samples. This can be further improved by considering the subjects involved in the entire region or environment.

\section{ACKNOWLEDGEMENTS}

The authors would like to thank Dr. Raveesh B N, Director of Dharwad Institute of Mental Health \& Neurosciences (DIMHANS) Dharwad, India for supporting the conduction of experiments in the current research work. Also authors extends thanks to Dr. T S Satyanarayana Rao, Professor and Head, Department of Psychiatry, JSS Medical College and Hospital, Mysusu, India for valuable guidelines.

\section{APPEAL}

This research work has been taken up to study and suggest an individual for their overall growth and developments in terms of leading happy life, not to heart or blame anybody in the environment or society.

\section{REFERENCES}

[1] L. Thurstone, “The mental age concept.” Psychological Review, vol. 33, no. 4, p. 268, 1926.

[2] R. Bar-On, R. Handley, and S. Fund, "The impact of emotional intelligence on performance," Linking emotional intelligence and performance at work: Current research evidence with individuals and groups, pp. 3-20, 2006.

[3] R. Bar-On, D. Tranel, N. L. Denburg, and A. Bechara, "Exploring the neurological substrate of emotional and social intelligence," Brain, vol. 126, no. 8, pp. 1790-1800, 2003.

[4] D. Goleman, "Working with emotional intelligence". Bantam, 1998.

[5] J. Mayer, "Msceit: Mayer-salovey-caruso emotional intelligence test," Toronto, Canada: Multi-Health Systems, 2002.

[6] A. S. Kaufman, "IQ testing 101“. Springer Publishing Company, 2009.

[7] A. K. Jain, "Data clustering: 50 years beyond k-means," Pattern recognition letters, vol. 31, no. 8, pp. 651-666, 2010.

[8] I. A. NeuroSky, "Thinkgear socket protocol (tech. rep.)," Available: http://www.neurosky.com/, 2010.

[9] Y. Renard, F. Lotte, G. Gibert, M. Congedo, E. Maby, V. Delannoy, O. Bertrand, and A. L'ecuyer, "Openvibe: An open-source software platform to design, test, and use brain-computer interfaces in real and virtual environments," Presence: teleoperators and virtual environments, vol. 19, no. 1, pp. 35-53, 2010.

[10] A. Azemi and C. Stook, "Utilizing matlab in undergraduate electric circuits courses," in Frontiers in Education Conference, 1996. FIE'96. 26th Annual Conference., Proceedings of, vol. 2. IEEE, 1996, pp.599-602.

[11] M. Inc., "Matlab users guide. [online]," Available: http://www.mathworks.com, 2012.

[12] S. Mouneshachari, M. S. Pande, and T. S. Rao, "Eq and iq based classification of intelligent index (s-quotient) using k-means," in Advanced Computing (IACC), 2016 IEEE 6th International Conference on. IEEE, 2016, pp. 101-105.

[13] A. M. Bihrle, H. H. Brownell, J. A. Powelson, and H. Gardner, "Comprehension of humorous and nonhumorous materials by left and right brain-damaged patients," Brain and Cognition, vol. 5, no. 4, pp. 399-411, 1986.

[14] M. Riemer et al., "IQ versus EQ: Emotional intelligence and the graduate engineer," in Proc. 5th Baltic Region Seminar on Engng. Educ, 2001, pp. 79-82.

[15] W. Ma, D. Tran, T. Le, H. Lin, and S.-M. Zhou, "Using eeg artifacts for bci applications," in Neural Networks (IJCNN), 2014 International Joint Conference on. IEEE, 2014, pp. 3628-3635.

[16] A. J. Casson, D. C. Yates, S. J. Smith, J. S. Duncan, and E. Rodriguez-Villegas, "Wearable electroencephalography," IEEE engineering in medicine and biology magazine, vol. 29, no. 3, pp. 44-56, 2010.

[17] Mouneshachari S, Dr. Sanjay Pande. M. B, and Dr. Raveesh. B. N, "Eeg Artifact Detection Model: A Landmark Based Approach," in Progress in Advanced Computing and Intelligent Engineering. Springer Nature, 2018.

[18] Dania, Ahmad, Abdul Rahman, and Hanim Hussin. "Detection of attention and meditation state-based brainwave system to control prosthetic arm." Indonesian Journal of Electrical Engineering and Computer Science (IJEECS) 13.2 (2019): 794-800.

[19] A. Forechi, A. F. De Souza, C. Badue, and T. Oliveira-Santos, "Sequential appearance-based global localization using an ensemble of knndtw classifiers," in Neural Networks (IJCNN), 2016 International Joint Conference on. IEEE, 2016, pp. 2782-2789.

[20] C. Zhu, R. Hou, and X. Ding, "An improved hybrid rfvim-knn method for high dimensional data," in Intelligent Human-Machine Systems and Cybernetics (IHMSC), 2016 8th International Conference on, vol. 2. IEEE, 2016, pp. 164-167.

[21] S. Rajathi and G. Radhamani, "Prediction and analysis of rheumatic heart disease using knn classification with aco," in Data Mining and Advanced Computing (SAPIENCE), International Conference on. IEEE, 2016, pp. 68-73. 
[22] Q. Wang and S. Ju, "A mixed classifier based on combination of hmm and knn," in Natural Computation, 2008. ICNC'08. Fourth International Conference on, vol. 4. IEEE, 2008, pp. 38-42.

[23] K. Machhale, H. B. Nandpuru, V. Kapur, and L. Kosta, "Mri brain cancer classification using hybrid classifier (svm-knn)," in Industrial Instrumentation and Control (ICIC), 2015 International Conference on. IEEE, 2015, pp. 60-65.

[24] J. H. AlKhateeb, F. Khelifi, J. Jiang, and S. S. Ipson, "A new approach for off-line handwritten arabic word recognition using knn classifier," in Signal and Image Processing Applications (ICSIPA), 2009 IEEE International Conference on. IEEE, 2009, pp. 191-194.

[25] Abbas F. H. Alharan, Hayder K. Fatlawi, Nabeel Salih Ali,"A cluster-based feature selection method for image texture classification", Indonesian Journal of Electrical Engineering and Computer Science (IJEECS), 14.3 (2019): $1433-1442$.

[26] L. J. Cronbach, "Coefficient alpha and the internal structure of tests," psychometrika, vol. 16, no. 3, pp. 297-334, 1951.

[27] A. Sarma, "Swami vivekananda's leadership formulas to become courageous," 2011.

\section{BIOGRAPHIES OF AUTHORS}

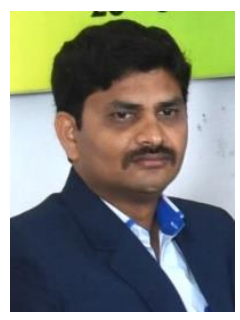

Dr. Mouneshachari S, Associate Professor in Department of Computer Science \& Engineering, GM Institute of Technology, Davangere, Karnataka, India. He has around sixteen years of experience in teaching and two years in Industry. He has completed his Ph.D. in Computer Science \& Engineering from Jain University, Bengaluru during 2017-18. His area of research includes Machine Learning in medical sciences, Robotics and Artificial Intelligence. The author has published many papers in National and International reputed publications. He is a technical reviewer for many International and National journals and conferences namely "IEEE Transactions on Signal Processing”, IEEE International Conference on Electrical, Electronics, Communication, Computer Technologies \& Optimization Techniques (ICEECCOT) and so on. The author also involved in many software, web and mobile application development activities.

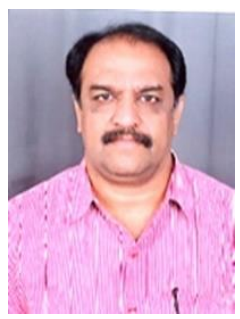

Dr. Sanjay Pande M B has done his PhD program in Developing Algorithmic models for neurological disorders, 12 students have been awarded Ph.D. under his supervision. His areas of interest are developing automation tool to Medical Data. He has a total of 97 publications under journal and conferences. He was awarded Young Scientist Award from KEELE UNIVERSITY, UK for his doctoral program. Has been instrumental in Initiating MITRA-Mental Informatics and Technical Research Academy, Mysore in collaboration with Dr. Raveesh B N, former Director, Dhimans, Dharwad. He is a reviewer for reputed International and National Journals. Author has a teaching experience of around 19 years. Recently he has started to give motivational / Inspirational speeches for Under Graduate and Post Graduate students. He is a FIE-Fellow of Institution of Engineers and also has several memberships from reputed professional bodies. 